\title{
Interval oscillation criteria for forced Emden-Fowler functional dynamic equations with oscillatory potential
}

\author{
ANDERSON Douglas R. ${ }^{1}$ \& SAKER Samir H. ${ }^{2, *}$ \\ ${ }^{1}$ Department of Mathematics and Computer Science, Concordia College, Moorhead, MN 56562, USA; \\ ${ }^{2}$ Department of Mathematics, Faculty of Science, Mansoura University, Mansoura 35516, Egypt \\ Email: andersod@cord.edu, shsaker@mans.edu.eg
}

Received April 25, 2011; accepted April 19, 2012; published online August 8, 2012

\begin{abstract}
Interval oscillation criteria are established for a second-order functional dynamic equation of EmdenFowler type with oscillatory potential by applying Riccati and generalized Riccati techniques. The results represent further improvements on those given even for differential and difference equations. Some examples are considered to illustrate the main results.
\end{abstract}

Keywords oscillation, forced dynamic equations, time scales, functional equations, Riccati technique

MSC(2010) 34K11, 39A10, 39A99

Citation: Anderson D R, Saker S H. Interval oscillation criteria for forced Emden-Fowler functional dynamic equations with oscillatory potential. Sci China Math, 2013, 56: 561-576, doi: 10.1007/s11425-012-4487-7

\section{Introduction}

In this paper, we are concerned with oscillation of the second-order nonlinear Emden-Fowler functional dynamic equation with oscillatory potential and forcing term on time scales of the form

$$
\left(r x^{\Delta}\right)^{\Delta}(t)+q(t)|x(\tau(t))|^{\gamma} x(\tau(t))=g(t), \quad t \in \mathbb{T},
$$

where $\mathbb{T}$ is a time scale unbounded above, $\gamma>1$, the potentials $r$ and $q$ and the forcing function $g$ are right-dense continuous with $r>0$, and $\tau: \mathbb{T} \rightarrow \mathbb{T}$ satisfies $\lim _{t \rightarrow \infty} \tau(t)=\infty$. Our interest is to establish some oscillation criteria for (1.1) that do not assume that $g$ and $q$ are of definite sign. When $\mathbb{T}=\mathbb{R}$, Equation (1.1) arises from a certain radial solution of the Klein-Gordon equation which is the relativistic version of the Schrödinger equation, and used to describe spinless particles.

Equation (1.1) is called a delay dynamic equation if $\tau(t)<t$, an advance dynamic equation, if $\tau(t)>t$, and an ordinary equation, if $\tau(t)=t$. As we are interested in the oscillatory and asymptotic behavior of solutions near infinity, we assume that $\sup \mathbb{T}=\infty$, and define the time scale interval $\left[t_{0}, \infty\right)_{\mathbb{T}}$ by $\left[t_{0}, \infty\right)_{\mathbb{T}}:=\left[t_{0}, \infty\right) \cap \mathbb{T}$. By a solution of (1.1) we mean that there exists a $t_{x} \geqslant t_{0}$ and a nontrivial realvalued function $x \in \mathrm{C}_{\mathrm{rd}}^{1}\left[t_{x}, \infty\right)_{\mathbb{T}}$ such that $x^{[1]}(t):=r(t) x^{\Delta}(t) \in \mathrm{C}_{\mathrm{rd}}^{1}\left[t_{x}, \infty\right)_{\mathbb{T}}$ and satisfies Equation (1.1) on $\left[t_{x}, \infty\right)_{\mathbb{T}}$, where $\mathrm{C}_{\mathrm{rd}}$ is the space of rd-continuous functions. The function $f: \mathbb{T} \rightarrow \mathbb{R}$ is said to be $r d$-continuous if it is continuous at each point and if there exists a finite left limit in all left-dense points. The solutions vanishing in some neighborhood of infinity will be excluded from our consideration.

${ }^{*}$ Corresponding author 
The study of dynamic equations on time scales, which goes back to its founder Hilger [15], is an area of mathematics that has recently received a lot of attention. It has been created in order to unify the study of differential and difference equations. Many results concerning differential equations carry over quite easily to corresponding results for difference equations, while other results seem to be completely different from their continuous counterparts. The study of dynamic equations on time scales reveals such discrepancies. The general idea is to prove a result for a dynamic equation where the domain of the unknown function is a so-called time scale $\mathbb{T}$. This way the results are not only related to the set of real numbers or the set of integers but those pertaining to more general time scales are obtained.

The three most popular examples of calculus on time scales are differential calculus, difference calculus, and quantum calculus (see Kac and Cheung [18]), i.e., when $\mathbb{T}=\mathbb{R}, \mathbb{T}=\mathbb{N}$, and $\mathbb{T}=q^{\mathbb{N}_{0}}=\left\{q^{t}: t \in \mathbb{N}_{0}\right\}$ where $q>1$. There are applications of dynamic equations on time scales to quantum mechanics, electrical engineering, neural networks, heat transfer, and combinatorics. A recent cover story article in New Scientist [29] discusses several possible applications. Since then, Bohner and Peterson [5] have expounded on various aspects of this new theory. The book on the subject of time scales, i.e., measure chains, by Bohner and Peterson [4] summarizes and organizes much of time scale calculus. For completeness, we recall the following concepts related to the notion of time scales. A time scale $\mathbb{T}$ is an arbitrary nonempty closed subset of the real numbers $\mathbb{R}$. The set of all such rd-continuous functions is denoted by $\mathrm{C}_{\mathrm{rd}}(\mathbb{T})$. The graininess function $\mu$ for a time scale $\mathbb{T}$ is defined by $\mu(t):=\sigma(t)-t$, and for any function $f: \mathbb{T} \rightarrow \mathbb{R}$ the notation $f^{\sigma}(t)$ denotes $f(\sigma(t))$.

We say that a solution $x$ of (1.1) has a generalized zero at $t$ if $x(t)=0$. We say that $x$ has a generalized zero in $(t, \sigma(t))$ in case $r(t) x(t) x^{\sigma}(t)<0$ and $\mu(t)>0$. Equation (1.1) is disconjugate on the interval $[a, b]_{\mathbb{T}}$, if there is no nontrivial solution of (1.1) with two (or more) generalized zeros in $[a, b]_{\mathbb{T}}$. Equation (1.1) is said to be nonosillatory on $[a, \infty)_{\mathbb{T}}$ if there exists $c \in[a, \infty)_{\mathbb{T}}$ such that this equation is disconjugate on $[c, d]_{\mathbb{T}}$ for every $d>c$. In the opposite case, $(1.1)$ is said to be oscillatory on $[a, \infty)_{\mathbb{T}}$.

The oscillation of the solutions of Equation (1.1) may equivalently be defined as follows: A nontrivial solution $x$ of (1.1) is called oscillatory if it has infinitely many (isolated) generalized zeros in $[a, \infty)_{\mathbb{T}}$; otherwise, it is called nonoscillatory. Equation (1.1) is said to be oscillatory if all its solutions are oscillatory. By the Sturm Separation Theorem, we see that oscillation is an interval property, i.e., if there exists a sequence of subintervals $\left[a_{i}, b_{i}\right]_{\mathbb{T}}$ of $\left[t_{0}, \infty\right)_{\mathbb{T}}$, as $i \rightarrow \infty$, such that for every $i$ there exists a solution of (1.1) that has at least two generalized zeros in $\left[a_{i}, b_{i}\right]_{\mathbb{T}}$, then every solution of (1.1) is oscillatory in $[a, \infty)_{\mathbb{T}}$. Hence we can speak about oscillation and nonoscillation of (1.1).

A great deal of effort has been spent in obtaining criteria for oscillation of equations without forcing terms, for example in the papers $[1,6,8-11,21-24]$ and the references cited therein. On the other hand, very little is known for related equations with forcing terms. In the following, we recall some of the related results that have been established for forced dynamic equations on time scales in $[7,13,16,17,26,27]$ that serve to motivate the contents of this paper. For more results of oscillation theory of dynamic equations on time scales, we refer the reader to the book by Saker [25].

Saker [26] considered the equation

$$
\left.x^{\Delta \Delta}(t)+q^{\sigma}(t)\right) f(x(t))=g(t), \quad t \in \mathbb{T},
$$

where $g$ and $q^{\sigma}$ are real-valued rd-continuous functions defined on the time scale $\mathbb{T}$ such that $q^{\sigma}>0$, and established some sufficient conditions which ensure that the solutions are bounded. Saker [27] considered the more general nonlinear forced dynamic equation

$$
\left(r(t) x^{\Delta}\right)^{\Delta}+q(t) f\left(x^{\sigma}\right)=g(t), \quad \text { for } t \in\left[t_{0}, \infty\right)_{\mathbb{T}},
$$

where $r(t)>0, q$ and $g$ are real-valued rd-continuous functions defined on the time scale $\mathbb{T}$. The author established some sufficient conditions for oscillation when the forcing term $g$ is small enough for large values of $t \in \mathbb{T}$. Bohner and Tisdell [7] considered (1.3) and established some sufficient conditions for oscillation when the unforced equation is nonoscillatory. The results depend on some additional assumptions imposed on the unknown solutions. Huang and Feng [16] considered the equation

$$
x^{\Delta \Delta}(t)+q(t) f\left(x^{\sigma}(t)\right)=g(t), \quad \text { for } t \in\left[t_{0}, \infty\right)_{\mathbb{T}},
$$


when $x f(x)>0$ for $x \neq 0$ and $|f(x)| \geqslant|x|^{\gamma}$ for $\gamma>1$, and established some interval criteria for oscillation based on the information on only a sequence of subintervals of $\left[t_{0}, \infty\right)_{\mathbb{T}}$. Huang and Feng [17] also considered (1.4) and applied a generalized Riccati technique introduced in [10] to establish some sufficient conditions for oscillation based on the information of $g$ and $q$ on only a sequence of subintervals of $\left[t_{0}, \infty\right)_{\mathbb{T}}$. Anderson [2] investigated the nonlinear second-order dynamic equation

$$
\left(r x^{\Delta}\right)^{\Delta}(t)+p(t)|x(\tau(t))|^{\alpha-1} x(\tau(t))+q(t)|x(\theta(t))|^{\beta-1} x(\theta(t))=f(t),
$$

for $t \in\left[t_{0}, \infty\right)_{\mathbb{T}}$ with $t_{0} \in \mathbb{T}$ and $t_{0} \geqslant 0$, where the parameters $\alpha, \beta \geqslant 1$, the functions $r, p, q, f: \mathbb{T} \rightarrow \mathbb{R}$ are rd-continuous with $r>0$ nondecreasing, the functions $\theta, \tau: \mathbb{T} \rightarrow \mathbb{T}$ are nondecreasing and rd-continuous back into the time scale with advance $\theta(t) \geqslant t$ and delay $\tau(t) \leqslant t$ such that $\tau(t) \rightarrow \infty$ as $t \rightarrow \infty$. These results are modeled after the continuous case given by Güvenilir and Zafer [14]. Anderson [3] also considered a more general nonlinear dynamic equation of the form

$$
\left(r x^{\Delta}\right)^{\Delta}(t)+q(t) f\left(t, x^{\sigma}, x^{\Delta}\right)=0, \quad t \in\left[t_{0}, \infty\right)_{\mathbb{T}}
$$

where $r(t)>0$ and $f: \mathbb{T} \times \mathbb{R} \rightarrow \mathbb{R}$ are rd-continuous functions on $\mathbb{T}$; he established some interval criteria for oscillation based on the information of $q$ on only a sequence of subintervals of $\left[t_{0}, \infty\right)_{\mathbb{T}}$. Erbe et al. [12] considered the second-order nonlinear dynamic equation of Emden-Fowler type with the forcing term

$$
\left(p(t) x^{\Delta}(t)\right)^{\Delta}+q(t)\left|x^{\sigma}(t)\right|^{\gamma} x^{\sigma}(t)=g(t),
$$

where $\gamma \geqslant 1, p>0, q>0$ and $g$ are rd-continuous functions, and established some sufficient conditions for oscillation based on the information of $g$ on only a sequence of subintervals of $\left[t_{0}, \infty\right)_{\mathbb{T}}$. Erbe et al. [13] considered the delay dynamic equation with the forcing term

$$
\left(r(t) x^{\Delta}(t)\right)^{\Delta}+q(t) f(x(\tau(t)))=g(t)
$$

on a time scale $\mathbb{T}$, where $g, r$, and $q$ are rd-continuous functions such that $r(t)>0, \tau(t) \leqslant t$ and $\lim _{t \rightarrow \infty} \tau(t)=\infty$. Under the assumption that the function $f \in \mathrm{C}(\mathbb{R}, \mathbb{R})$ satisfies $u f(u)>0$ for $u \neq 0$, and $|f(u)| \geqslant k|u|$ for some $k>0$, their criteria again were based on the information of $g$ and $q$ on only a sequence of subintervals of $\left[t_{0}, \infty\right]_{\mathbb{T}}$.

Note that (1.1) in its general form involves some different types of differential and difference equations depending on the choice of the time scale $\mathbb{T}$. For example, when $\mathbb{T}=\mathbb{R},(1.1)$ includes the second-order delay differential equations

$$
x^{\prime \prime}(t)+q(t) x^{\gamma}(\tau(t)) x(\tau(t))=g(t), \quad t \geqslant t_{0},
$$

and

$$
\left(r x^{\prime}\right)^{\prime}+q(t) x^{\gamma}(\tau(t)) x(\tau(t))=g(t), \quad t \geqslant t_{0} .
$$

Nasr [19] considered (1.8) and extended the results by Wong [32] and proved the following result: If $\gamma>1, q(t)>0$, and there exists a bounded function $h$ such that $h^{\prime \prime}(t)=g(t), \tau^{\prime \prime}(t)>\alpha>0$ and

$$
\int_{t_{0}}^{\infty} t q(t) d t=\infty,
$$

then every solution of (1.8) is oscillatory. Note that the results given by Nasr [19] are based on the information of the coefficients on the whole half-line $\left[t_{0}, \infty\right]$ when the potential is a positive function. On the other hand, one can easily see that the condition (1.9) is not sharp and cannot be applied to some equations. For example, if we consider the equation

$$
x^{\prime \prime}(t)+\frac{\lambda}{t^{2}} x(t)=5+4 \sin (\ln t)+3 \cos (\ln t),
$$

we see that the condition (1.9) is satisfied for any positive value of $\lambda$. But the equation is not oscillatory when $\lambda=2$ and when $\lambda=3$, Equation (1.10) is oscillatory. Thus, the results that have been established by Wong and Nasr are not sharp. Wang [31] considered the equation

$$
\left(r x^{\prime}\right)^{\prime}(t)+q(t) f\left(t, x, x^{\prime}\right)=0, \quad t \geqslant t_{0},
$$


where $r>0, f: \mathrm{C}\left[t_{0}, \infty\right) \times \mathbb{R}^{2} \rightarrow \mathbb{R}$, and established some interval criteria for oscillation based on the information of $q$ on only a sequence of subintervals of $\left[t_{0}, \infty\right)$. The results in [3] can be considered as the time scale analogue of the results that have been established in [31]. Sun [30] considered Equation (1.7) and extended the results established by Wong [32] and Nasr [19] for the forced equation without delay, and established some interval criteria for oscillation.

When $\mathbb{T}=\mathbb{Z}$, we have $\sigma(t)=t+1, \mu(t)=1, f^{\Delta}(t)=\Delta f(t), \int_{a}^{b} f(t) \Delta t=\sum_{t=a}^{b-1} f(t)$, and (1.1) includes the difference equations

$$
\begin{aligned}
& \Delta(r(t) \Delta x(t))+q(t)|x(t)|^{\gamma} x(t)=g(t), \\
& \Delta^{2} x(t)+q(t)|x(t)|^{\gamma} x(t)=g(t) .
\end{aligned}
$$

Saker [28] considered (1.11) and established some sufficient conditions for oscillation of Kamenev type. The results in [28] depend on the information of the coefficients on the whole half-line $\left[t_{0}, \infty\right]$. Note that the results that have been established in [28] cannot be applied to the Emden-Fowler equations with delay.

We notice that the main challenge in the oscillation theory of differential and difference equations with coefficients of no fixed signs, equations with damping terms and equations with oscillatory potential and forced terms is the determination of the sign of the first derivative of the solution. In fact, in differential equations (or difference equations) if one knows that the solution $x(t)$ (or the sequence $x(n)$ ) is defined on an interval $\left[t_{0}, \infty\right)\left(\right.$ or $\left.\left[n_{0}, \infty\right)\right)$ and satisfies $x(t)>0($ or $x(n)>0)$ and $x^{\prime \prime}(t)<0$ (or $\left.\Delta^{2} x(n)<0\right)$ for all $t \geqslant t_{0}$, (or $\left.n \geqslant n_{0}\right)$ then $x^{\prime}(t)>0$ (or $\Delta x(n)>0$ ) for all $t>t_{1} \geqslant t_{0}$ (or $n>n \geqslant n_{0}$ ). Geometrically, this means that a positive concave down function must be eventually increasing (i.e., there exists a $T>t_{0}$ sufficiently large such that $x^{\prime}(t)>0$ for $t \geqslant T$ ). These facts are very often used in the oscillation theory of differential and difference equations. The same conclusion is true when the derivative or the difference is replaced by a $\Delta$-derivative on a time scale and also often used in oscillation of dynamic equations on time scales (see [25]). But if $x(t)>0$ and $x^{\prime \prime}(t)<0$ only on a sequence of intervals then $x^{\prime}(t)>0$ is not necessarily true on the intervals. The same conclusion is true when the derivative is replaced by a $\Delta$-derivative on a time scale. In this paper, we will overcome this challenge and use a new approach to establish some oscillation criteria for Equation (1.1) without any information about the sign of $x^{\Delta}(t)$.

The main results will be proved in Section 2 which is organized as follows: In Subsection 2.1, we will prove some oscillation results which cover the case $\tau(t)>t$. In Subsection 2.2, we will prove some results which cover the case $\tau(t) \leqslant t$. The special case when $r(t)=1$ and $\tau(t)=\sigma(t)$ include the results that have been established in [16, Theorem 3.2], and when $\tau(t)=t$ our results include the results established in [3, Corollary 3.4], and when $\tau(t)=\sigma(t)$ our results improve the results established in [12, Theorem 3.2] in the sense that our results do not require that $q>0$ on $\left[t_{0}, \infty\right)_{\mathbb{T}}$. When $\mathbb{T}=\mathbb{R}$ the results improve the results by Nasr [19, Theorem 2] and involve the results established by Sun [30, Theorem 1] and Wang [31, Corollary 2.5]. When $\mathbb{T}=\mathbb{N}$, our results improve the oscillation results established by Saker [28, Theorem 2.1] in the sense that our results are based on the information of $g$ and $q$ on only a sequence of subintervals of $\left[t_{0}, \infty\right]_{\mathbb{T}}$. Some examples are given to illustrate the main results.

\section{Main results}

In this section, by using the Riccati transformation techniques (Riccati substitution and generalized Riccati substitution) we will establish some interval oscillation criteria, that is, criteria given by the behavior of $g$ and $q$ only on a sequence of subintervals of $\left[t_{0}, \infty\right)_{\mathbb{T}}$. We define

$$
\mathbb{D}:=\left\{u: u \in \mathrm{C}_{\mathrm{rd}}^{1}\left(\left[s_{i}, t_{i}\right]\right), u(t) \neq 0, \text { and } u\left(s_{i}\right)=u\left(t_{i}\right)=0\right\}, \quad i=1,2 .
$$




\subsection{The case when $\tau(t)>t$}

In this subsection, we establish some sufficient conditions for oscillation of $(1.1)$ when $\tau(t)>t$. We introduce the auxiliary function

$$
Q_{i}(t):=\gamma(\gamma-1)^{\frac{1}{\gamma}-1}(q(t))^{1 / \gamma} C_{i}\left(t, s_{i}\right)|g(t)|^{1-\frac{1}{\gamma}},
$$

where for $i=1,2$,

$$
C_{i}\left(t, s_{i}\right):= \begin{cases}\frac{R\left(s_{i}\right)-R(\tau(t))}{R\left(s_{i}\right)-R(\sigma(t))}, & \text { if } \tau(t)>\sigma(t), \\ 1, & \text { if } \tau(t)=\sigma(t),\end{cases}
$$

and

$$
R(t):=\int^{t} \frac{1}{r(s)} \Delta s>0, \quad R\left(t, s_{1}\right)=R(t)-R\left(s_{1}\right)=\int_{s_{1}}^{t} \frac{1}{r(s)} \Delta s>0 .
$$

Note that (2.1) is different from (3.10) and (3.11) in [2]. We assume that the following condition holds:

$\left(\mathrm{H}_{1}\right)$ Given any $T \in\left[t_{0}, \infty\right)_{\mathbb{T}}$ there exist points $T<s_{i}<t_{i}$ in $\mathbb{T}$ for $i=1,2$ such that $q \geqslant 0$ for $t \in\left[s_{1}, t_{1}\right)_{\mathbb{T}} \cup\left[s_{2}, t_{2}\right)_{\mathbb{T}}$ and

$$
g(t)\left\{\begin{array}{l}
\leqslant 0: t \in\left[s_{1}, t_{1}\right)_{\mathbb{T}}, \\
\geqslant 0: t \in\left[s_{2}, t_{2}\right)_{\mathbb{T}} .
\end{array}\right.
$$

Theorem 2.1. $\quad$ Assume that $(2.3)$ and $\left(\mathrm{H}_{1}\right)$ hold and $\tau(t)>t$. Further assume that there exists an $r d$-continuous function $u \in \mathbb{D}$ such that for $i=1$, 2 we have

$$
A_{i}(u):=\int_{s_{i}}^{t_{i}}\left[r(t)\left(u^{\Delta}(t)\right)^{2}-Q_{i}(t)\left(u^{\sigma}(t)\right)^{2}\right] \Delta t \leqslant 0 .
$$

Then (1.1) is oscillatory.

Proof. Assume that there is a nonoscillatory solution $x$ of (1.1). First, we consider the case $x(t)>0$ and $x(\tau(t))>0$ for $t \geqslant T$. We make the Riccati substitution

$$
w(t):=-\frac{x^{[1]}(t)}{x(t)}, \quad t \in[T, \infty)_{\mathbb{T}} .
$$

Then, suppressing the argument, we have

$$
\begin{aligned}
w^{\Delta} & =\frac{x\left(-r x^{\Delta}\right)^{\Delta}+r\left(x^{\Delta}\right)^{2}}{x x^{\sigma}}=q \frac{(x(\tau))^{\gamma}-g}{x^{\sigma}}+\frac{r\left(x^{\Delta}\right)^{2}}{x x^{\sigma}} \\
& =q \frac{(x(\tau))^{\gamma}}{x^{\sigma}}-\frac{g}{x^{\sigma}}+\frac{w^{2} x}{r x^{\sigma}}=q \frac{(x(\tau))^{\gamma}}{x^{\sigma}}-\frac{g}{x^{\sigma}}+\frac{w^{2} x}{r\left(x+\mu x^{\Delta}\right)} \\
& =q \frac{(x(\tau))^{\gamma}}{x^{\sigma}}-\frac{g}{x^{\sigma}}+\frac{w^{2}}{r\left(1+\frac{\mu x^{\Delta}}{x}\right)}=q \frac{(x(\tau))^{\gamma}}{x^{\sigma}}-\frac{g}{x^{\sigma}}+\frac{w^{2}}{r-\mu w} .
\end{aligned}
$$

Since

$$
0<\frac{x}{r x^{\sigma}}=\frac{x}{r\left(x+\mu x^{\Delta}\right)}=\frac{1}{r-\mu w},
$$

we see that $r-\mu w>0$. Therefore, we have

$$
w^{\Delta}=q\left(\frac{x(\tau)}{x^{\sigma}}\right)^{\gamma}\left(x^{\sigma}\right)^{\gamma-1}-\frac{g}{x^{\sigma}}+\frac{w^{2}}{r-\mu w} .
$$

By the assumptions, we can choose $t_{1}>s_{1}>T$ such that $q(t) \geqslant 0$ for $t \in\left[s_{1}, t_{1}\right)_{\mathbb{T}}$, and $g(t) \leqslant 0$ for $t \in\left[s_{1}, t_{1}\right)_{\mathbb{T}}$. Then from (1.1), we have

$$
\left(x^{[1]}\right)^{\Delta}=-q(t) x^{\gamma}(\tau(t))+g(t) \leqslant 0, \quad \text { for } t \in\left[s_{1}, t_{1}\right)_{\mathbb{T}} .
$$


Hence $x^{[1]}=r x^{\Delta}$ is nonincreasing for $t \in\left[s_{1}, t_{1}\right)_{\mathbb{T}}$. Next, consider the coefficient of $q$ in $(2.6)$. Set

$$
z(t)=x^{[1]}(t)= \begin{cases}r(t) x^{\Delta}(t), & \text { if } x^{\Delta}(t) \geqslant 0 \\ r(t)\left(-x^{\Delta}(t)\right), & \text { if } x^{\Delta}(t)<0\end{cases}
$$

Then $z(t)$ is an rd-continuous function defined on $\left[s_{1}, t_{1}\right)_{\mathbb{T}}$. Since $x^{[1]}$ is nonincreasing for $t \in\left[s_{1}, t_{1}\right)_{\mathbb{T}}$, we have that $z(t)$ is a nonincreasing function in $\left[s_{1}, t_{1}\right)_{\mathbb{T}}$. Noting that $x^{\Delta}=r^{-1}(t) z(t)$, we have

$$
\begin{aligned}
0 & <x(t)=x\left(s_{1}\right)+\int_{s_{1}}^{t} x^{\Delta}(s) \Delta s \\
& =x\left(s_{1}\right)+\int_{s_{1}}^{t} z(s) \frac{1}{r(s)} \Delta s \geqslant z(t) \int_{s_{1}}^{t} \frac{1}{r(s)} \Delta s \\
& =\left[R(t)-R\left(s_{1}\right)\right] r(t) x^{\Delta}(t) .
\end{aligned}
$$

Now, we define $U(t)$ by

$$
U(t):=x(t)-\left[R(t)-R\left(s_{1}\right)\right]\left(r(t) x^{\Delta}(t)\right), \quad t \in\left[s_{1}, t_{1}\right)_{\mathbb{T}} .
$$

From (2.3) and (2.7) we have

$$
\begin{aligned}
U^{\Delta}(t) & =-R\left(\sigma(t), s_{1}\right)\left(r x^{\Delta}\right)^{\Delta}(t) \\
& =R\left(\sigma(t), s_{1}\right) q(t) x^{\gamma}(\tau(t))-R(\sigma(t)) g(t)>0
\end{aligned}
$$

for $t \in\left[s_{1}, t_{1}\right)_{\mathbb{T}}$, and this implies that $U(t)$ is strictly increasing on $\left[s_{1}, t_{1}\right)_{\mathbb{T}}$. Also by $(2.9)$, we see that $U(t) \geqslant 0$ for $s \in[t, \tau(t)]$ and $t \in\left[s_{1}, t_{1}\right)_{\mathbb{T}}$. From $(2.10)$ and $(2.3)$, we see that

$$
\begin{aligned}
0 \leqslant \frac{r^{-1}(t) U(t)}{x(t) x^{\sigma}(t)} & :=\frac{r^{-1}(t)}{x^{\sigma}(t)}-\frac{R(t)-R\left(s_{1}\right)}{x(t) x^{\sigma}(t)} x^{\Delta}(t) \\
& =\left(\frac{R(t)-R\left(s_{1}\right)}{x(t)}\right)^{\Delta}, \quad \text { for } t \in\left[s_{1}, t_{1}\right)_{\mathbb{T}} .
\end{aligned}
$$

This implies that

$$
\begin{aligned}
0 & \leqslant \int_{t}^{\tau(t)} \frac{r^{-1}(s) U(s)}{x(s) x^{\sigma}(s)} \Delta s=\int_{t}^{\tau(t)}\left(\frac{R(s)-R\left(s_{1}\right)}{x(s)}\right)^{\Delta} \Delta s \\
& =\frac{R^{\tau}(t)-R\left(s_{1}\right)}{x^{\tau}(t)}-\frac{R(t)-R\left(s_{1}\right)}{x(t)},
\end{aligned}
$$

and then we get that

$$
\frac{x^{\tau}(t)}{x(t)} \geqslant \frac{R\left(s_{1}\right)-R(\tau(t))}{R\left(s_{1}\right)-R(t)} .
$$

Also, we get that

$$
\int_{t}^{\sigma(t)}\left(\frac{\left[R(t)-R\left(s_{1}\right)\right]}{x(s)}\right)^{\Delta} \Delta s=\frac{R^{\sigma}(t)-R\left(s_{1}\right)}{x^{\sigma}(t)}-\frac{R(t)-R\left(s_{1}\right)}{x(t)} \geqslant 0,
$$

and then

$$
\frac{x(t)}{x^{\sigma}(t)} \geqslant \frac{R(t)-R\left(s_{1}\right)}{R(\sigma(t))-R\left(s_{1}\right)} .
$$

From (2.11) and (2.12), we have

$$
\frac{x^{\tau}(t)}{x^{\sigma}(t)} \geqslant C_{1}\left(t, s_{1}\right)=\frac{R\left(s_{1}\right)-R(\tau(t))}{R\left(s_{1}\right)-R(\sigma(t))}, \quad \text { for } t \geqslant s_{1} .
$$

As a result,

$$
\left(\frac{x^{\tau}(t)}{x^{\sigma}(t)}\right)^{\gamma} \geqslant C_{1}^{\gamma}\left(t, s_{1}\right)
$$


Substituting from (2.14) into (2.6), we get

$$
w^{\Delta} \geqslant q(t) C_{1}^{\gamma}\left(t, s_{1}\right)\left(x^{\sigma}\right)^{\gamma-1}+\frac{|g(t)|}{x^{\sigma}}+\frac{w^{2}(t)}{r(t)-\mu(t) w(t)} .
$$

Using the fact that the function

$$
G(y):=a y^{\gamma-1}+\frac{b}{y}
$$

satisfies

$$
G(y) \geqslant \gamma(\gamma-1)^{\frac{1}{\gamma}-1} a^{\frac{1}{\gamma}} b^{1-1 / \gamma}, \quad \text { for } y>0
$$

we have

$$
\inf _{u>0}\left[q(t) C_{1}^{\gamma}\left(t, \tau\left(s_{1}\right)\right)\left(x^{\sigma}\right)^{\gamma-1}+\frac{|g|}{x^{\sigma}}\right] \geqslant Q_{1}(t) .
$$

Hence

$$
w^{\Delta} \geqslant Q_{1}(t)+\frac{w^{2}}{r-\mu w}, \quad \text { for } t \in\left[s_{1}, t_{1}\right]_{\mathbb{T}} .
$$

Now multiply by $\left(u^{\sigma}\right)^{2}$, where $u \in \mathbb{D}$ and integrate to get

$$
\int_{s_{1}}^{t_{1}}\left(u^{\sigma}\right)^{2} w^{\Delta} \Delta t \geqslant \int_{s_{1}}^{t_{1}} Q_{1}\left(u^{\sigma}\right)^{2} \Delta t+\int_{s_{1}}^{t_{1}} \frac{w^{2}\left(u^{\sigma}\right)^{2}}{r-\mu w} \Delta t .
$$

Rearranging and using $u\left(s_{1}\right)=0=u\left(t_{1}\right)$, we get (suppressing arguments)

$$
\begin{aligned}
0 & \geqslant \int_{s_{1}}^{t_{1}} \frac{w^{2}\left(u^{\sigma}\right)^{2}}{r-\mu w} \Delta t-\int_{s_{1}}^{t_{1}}\left[u+u^{\sigma}\right] u^{\Delta} w \Delta t+\int_{s_{1}}^{t_{1}} Q_{1}(t)\left(u^{\sigma}\right)^{2} \Delta t \\
& =\int_{s_{1}}^{t_{1}} \frac{w^{2}\left(u^{\sigma}\right)^{2}}{r-\mu w} \Delta t-\int_{s_{1}}^{t_{1}}\left[2 u^{\sigma} u^{\Delta} w-\mu\left(u^{\Delta}\right)^{2} w\right] \Delta t+\int_{s_{1}}^{t_{1}} Q_{1}(t)\left(u^{\sigma}\right)^{2} \Delta t .
\end{aligned}
$$

Adding and subtracting the term $\int_{s_{1}}^{t_{1}} r\left(u^{\Delta}\right)^{2} \Delta t$ and using the assumption $-A_{1}(u) \geqslant 0$, we have

$$
\begin{aligned}
0 \geqslant & \int_{s_{1}}^{t_{1}}\left(\frac{w^{2}\left(u^{\sigma}\right)^{2}}{r-\mu w}-2 u^{\sigma} u^{\Delta} w+[r+\mu w]\left(u^{\Delta}\right)^{2}\right) \Delta t \\
& -\int_{s_{1}}^{t_{1}}\left[r\left(u^{\Delta}\right)^{2}-Q_{1}(t)\left(u^{\sigma}\right)^{2}\right] \Delta t \\
= & \int_{s_{1}}^{t_{1}}\left(\frac{w u^{\sigma}}{\sqrt{r-\mu w}}-\sqrt{r+\mu w} u^{\Delta}\right)^{2} \Delta t-A_{1}(u) \\
\geqslant & \int_{s_{1}}^{t_{1}}\left(\frac{w u^{\sigma}}{\sqrt{r-\mu w}}-\sqrt{r-\mu w} u^{\Delta}\right)^{2} \Delta t .
\end{aligned}
$$

It follows that

$$
\int_{s_{1}}^{t_{1}}\left(\frac{w u^{\sigma}}{\sqrt{r-\mu w}}-\sqrt{r+\mu w} u^{\Delta}\right)^{2} \Delta t=0 .
$$

This implies that

$$
w(t) u^{\sigma}(t)-(r(t)-\mu(t) w(t)) u^{\Delta}(t)=0, \quad \text { for } \quad t \in\left[s_{1}, t_{1}\right)_{\mathbb{T}} .
$$

Solving for $u^{\Delta}$, we get that $u$ solves the IVP

$$
u^{\Delta}=\frac{w}{r+\mu w} u^{\sigma}, \quad u\left(s_{1}\right)=0, \quad \text { for } t \in\left[s_{1}, t_{1}\right)_{\mathbb{T}} .
$$

Since $-w /(r+\mu w) \neq 0$, we get from $\left[4\right.$, Theorem 2.71] that $u(t) \equiv 0$ on $\left[s_{1}, t_{1}\right)_{\mathbb{T}}$, which is a contradiction. The case $x(t)<0, x(\tau(t))<0$ on $[T, \infty)_{\mathbb{T}}$ is similar, where we use $g(t) \geqslant 0$ on $\left[\tau\left(s_{2}\right), t_{2}\right)_{\mathbb{T}}$ and $A_{2}(u) \leqslant 0$. The proof is completed. 
We note that the results in Theorem 2.1 depend on the test function $u$, enabling us to establish different sufficient conditions for the oscillation of (1.1) by using different choices of $u$. For instance, consider $t_{i}=s_{i}+\pi \sqrt{\alpha_{i}}, i=1,2$ where $s_{i}$ and $t_{i}$ are defined as in Theorem 2.1. This determines $\alpha_{i}$ for $i=1,2$. If we choose $u(t)=\sin \sqrt{\alpha_{i}}\left(t-s_{i}\right)$ and note $\left[4\right.$, Lemma 3.26] that $u^{\Delta}(t)=\sqrt{\alpha_{i}} \cos \sqrt{\alpha_{i}}\left(t-s_{i}\right)$, we have the following result:

Corollary 2.2. Assume that (2.3) and $\left(\mathrm{H}_{1}\right)$ hold and $\tau(t)>t$. Furthermore, assume for $\alpha_{i}=$ $\pi^{2} /\left(t_{i}-s_{i}\right)^{2}, i=1,2$ that

$$
\int_{s_{i}}^{t_{i}}\left[r(t) \alpha_{i} \cos ^{2} \sqrt{\alpha_{i}}\left(t-s_{i}\right)-Q_{i}(t) \sin ^{2} \sqrt{\alpha_{i}}\left(\sigma(t)-s_{i}\right)\right] \Delta t \leqslant 0 .
$$

Then (1.1) is oscillatory.

Another method of choosing test functions can be developed by considering the function class $\Re$ which consists of kernels of two variables. Following Saker [20], we say that the function $H \in \Re$ provided that $H$ is defined for $t_{0} \leqslant s \leqslant t$ for $t, s \in\left[t_{0}, \infty\right)_{\mathbb{T}}, H(t, s) \geqslant 0, H(t, t)=0$ for $t \geqslant s \geqslant t_{0}$, and for each fixed $t$, $H^{\Delta_{i}}(t, s)$ is delta integrable with respect to variable $i(i=1,2)$. Important examples of $H$ when $\mathbb{T}=\mathbb{R}$ are $H(t, s)=(t-s)^{m}$ for $m \geqslant 1$. When $\mathbb{T}=\mathbb{Z}, H(t, s)=(t-s)^{\underline{k}}, k \in \mathbb{N}$, where $t^{\underline{k}}=t(t-1) \cdots(t-k+1)$. For any $H_{1}$ and $H_{2} \in \Re$, if we let $u(t)=H_{1}\left(t_{1}, t\right) H_{2}\left(t, s_{1}\right)$, then it is easy to see that $u\left(s_{1}\right)=u\left(t_{1}\right)=0$, $u(t) \neq 0$ on $\left[s_{1}, t_{1}\right]$. Therefore, by choosing appropriate functions $H_{1}$ and $H_{2}$ we can derive oscillation criteria for (1.1) from Theorem 2.1. For a given function $H(t, s)$, we define $h_{1}(t, s)$ and $h_{2}(t, s)$ as follows:

$$
h_{1}\left(t, s_{1}\right)=\frac{H^{\Delta_{1}}\left(t, s_{1}\right)}{\sqrt{H\left(t, s_{1}\right)}} \quad \text { and } \quad h_{2}\left(t_{1}, t\right)=\frac{H^{\Delta_{2}}\left(t_{1}, t\right)}{\sqrt{H\left(t_{1}, t\right)}} .
$$

When $u(t)=H\left(t_{1}, t\right) H\left(t, s_{1}\right)$, we have

$$
\begin{aligned}
u^{\Delta}(t) & =\left(H\left(t_{1}, t\right) H\left(t, s_{1}\right)\right)^{\Delta}=H\left(t_{1}, t\right) H^{\Delta_{1}}\left(t, s_{1}\right)+H^{\Delta_{2}}\left(t_{1}, t\right) H\left(\sigma(t), s_{1}\right) \\
& =h_{1}\left(t, s_{1}\right) H\left(t_{1}, t\right) \sqrt{H\left(t, s_{1}\right)}+h_{2}\left(t_{1}, t\right) H\left(\sigma(t), s_{1}\right) \sqrt{H\left(t_{1}, t\right)} \\
& =\frac{h_{1}\left(t, s_{1}\right)}{\sqrt{H\left(t, s_{1}\right)}} u(t)+h_{2}\left(t_{1}, t\right) \frac{u^{\sigma} \sqrt{H\left(t_{1}, t\right)}}{H\left(t_{1}, \sigma(t)\right)} .
\end{aligned}
$$

Then from Theorem 2.1, we have the following result:

Corollary 2.3. Assume that (2.3) and $\left(\mathrm{H}_{1}\right)$ hold and $\tau(t)>t$. Furthermore, assume for $i=1,2$ that

$$
\int_{s_{i}}^{t_{i}}\left[r(t) Z_{i}^{2}-Q_{i}(t) H^{2}\left(t_{i}, \sigma(t)\right) H^{2}\left(\sigma(t), s_{i}\right)\right] \Delta t \leqslant 0,
$$

where $Z_{i}=h_{i}\left(t, s_{i}\right) H\left(t_{i}, t\right) \sqrt{H\left(t, s_{i}\right)}+h_{i}\left(t_{1}, t\right) H\left(\sigma(t), s_{i}\right) \sqrt{H\left(t_{i}, t\right)}$. Then (1.1) is oscillatory.

In the following, we employ a generalized Riccati transformation technique and establish some sufficient interval conditions for oscillation. We define

$$
\overline{C_{i}}\left(t, s_{1}\right)=\frac{R(t)-R\left(s_{i}\right)}{R^{\sigma}(t)-R\left(s_{i}\right)} .
$$

Theorem 2.4. Assume that (2.3) and $\left(\mathrm{H}_{1}\right)$ hold and $\tau(t)>t$. Furthermore, assume that there exist functions $a_{i}$ for $i=1,2$ and a positive, differentiable function $\delta$ and an $r d$-continuous function $u \in \mathbb{D}$ such that for $i=1,2$,

$$
\int_{s_{i}}^{t_{i}}\left(u^{\sigma}\right)^{2}\left[\psi_{i}-\frac{r \delta^{2}}{4 \delta^{\sigma} \overline{C_{i}}}\left(\left[\frac{\delta^{\Delta}}{\delta}+\frac{2 \delta^{\sigma} a_{i} \overline{C_{i}}}{\delta}\right]-\frac{\left[u+u^{\sigma}\right] u^{\Delta}}{\left(u^{\sigma}\right)^{2}}\right)^{2}\right] \Delta t>0,
$$

where $\overline{C_{i}}\left(t, s_{i}\right)$ is defined as in $(2.17)$ and

$$
\psi_{i}:=\delta^{\sigma}\left[Q_{i}-\left(a_{i} r\right)^{\Delta}+C_{i}\left(t, s_{i}\right) r a_{i}^{2}\right] .
$$

Then (1.1) is oscillatory. 
Proof. Suppose to the contrary that $x$ is a nonoscillatory solution of (1.1). Then there is a $t_{1} \in\left[t_{0}, \infty\right)_{\mathbb{T}}$ such that $x(\tau(t)) \neq 0$ on $\left[t_{1}, \infty\right)_{\mathbb{T}}$. We will only consider the case where $x(\tau(t))>0$ for all $t \in\left[t_{1}, \infty\right)_{\mathbb{T}}$. Define the function $w$ by the generalized Riccati substitution

$$
w_{1}(t):=\delta(t)\left[\frac{x^{[1]}(t)}{x(t)}+r(t) a_{1}(t)\right], \quad t \in\left[s_{1}, t_{1}\right] .
$$

Hence

$$
\begin{aligned}
w_{1}^{\Delta} & =\delta^{\Delta}\left[\frac{x^{[1]}}{x}+r a_{1}\right]+\delta^{\sigma}\left[\frac{x^{[1]}}{x}+r a_{1}\right]^{\Delta} \\
& =\frac{\delta^{\Delta}}{\delta} w_{1}+\delta^{\sigma}\left(r a_{1}\right)^{\Delta}+\delta^{\sigma}\left[\frac{x^{[1]}}{x}\right]^{\Delta} \\
& =\frac{\delta^{\Delta}}{\delta} w_{1}+\delta^{\sigma}\left(r a_{1}\right)^{\Delta}+\delta^{\sigma}\left[\frac{x\left(x^{[1]}\right)^{\Delta}-r\left(x^{\Delta}\right)^{2}}{x x^{\sigma}}\right] \\
& =\frac{\delta^{\Delta}}{\delta} w_{1}+\delta^{\sigma}\left(r a_{1}\right)^{\Delta}+\delta^{\sigma} \frac{\left(x^{[1]}\right)^{\Delta}}{x^{\sigma}}-\delta^{\sigma} \frac{r\left(x^{\Delta}\right)^{2}}{x x^{\sigma}} .
\end{aligned}
$$

This implies that

$$
w_{1}^{\Delta}=-\frac{\delta^{\sigma}}{x^{\sigma}}\left[q\left(\frac{x(\tau)}{x^{\sigma}}\right)^{\gamma}\left(x^{\sigma}\right)^{\gamma}+|g(t)|\right]+\frac{\delta^{\Delta}}{\delta} w_{1}+\delta^{\sigma}\left(r a_{1}\right)^{\Delta}-\delta^{\sigma} r \frac{\left(x^{\Delta}\right)^{2}}{x x^{\sigma}} .
$$

Using the inequality $a_{1} u^{\gamma}+b \geqslant \gamma(\gamma-1)^{\frac{1}{\gamma}-1} a_{1}^{1 / \gamma} b^{1-\frac{1}{\gamma}}$, with

$$
a_{1}=q\left(\frac{x(\tau)}{x^{\sigma}}\right)^{\gamma}>0, \quad b=|g(t)|>0, \quad \text { and } \quad u=x^{\sigma},
$$

we get by using (2.13) that

$$
\begin{aligned}
{\left[q\left(\frac{x(\tau)}{x^{\sigma}}\right)^{\gamma}\left(x^{\sigma}\right)^{\gamma}+|g(t)|\right] } & \geqslant \gamma(\gamma-1)^{\frac{1}{\gamma}-1} \frac{x(\tau)}{x^{\sigma}} q^{\frac{1}{\gamma}}(|g(t)|)^{1-\frac{1}{\gamma}} x^{\sigma} \\
& \geqslant \gamma(\gamma-1)^{1 / \gamma-1} q^{\frac{1}{\gamma}}(|g(t)|)^{1-\frac{1}{\gamma}} C_{1} .
\end{aligned}
$$

Substituting (2.23) into (2.22), we have

$$
w_{1}^{\Delta} \leqslant-\delta^{\sigma} Q_{1}(t)+\frac{\delta^{\Delta}}{\delta} w_{1}+\delta^{\sigma}\left(r a_{1}\right)^{\Delta}-\delta^{\sigma} r\left(\frac{x^{\Delta}}{x}\right)^{2} \frac{x}{x^{\sigma}} .
$$

From the definition of $w_{1}(t)$, we see that

$$
\left(\frac{x^{\Delta}}{x}\right)^{2}=\left[\frac{w_{1}}{r \delta}-a_{1}\right]^{2}=\left[\frac{w_{1}}{r \delta}\right]^{2}+a_{1}^{2}-2 \frac{w_{1} a_{1}}{r \delta} .
$$

Substituting (2.25) into (2.24), and using (2.12), we obtain

$$
\begin{aligned}
w_{1}^{\Delta} & \leqslant-\delta^{\sigma} Q_{1}(t)+\frac{\delta^{\Delta}}{\delta} w_{1}+\delta^{\sigma}\left(r a_{1}\right)^{\Delta}-\delta^{\sigma} r \overline{C_{1}}\left[\left[\frac{w_{1}}{r \delta}\right]^{2}+a_{1}^{2}-2 \frac{a_{1} w_{1}}{r \delta}\right] \\
& =-\delta^{\sigma} Q_{1}(t)+\frac{\delta^{\Delta}}{\delta} w_{1}+\delta^{\sigma}\left(r a_{1}\right)^{\Delta}-\frac{\overline{C_{1}} \delta^{\sigma} w_{1}^{2}}{r \delta^{2}}-\delta^{\sigma} r \overline{C_{1}} a_{1}^{2}+2 \frac{\delta^{\sigma} \overline{C_{1}} a_{1}}{\delta} w_{1} \\
& =-\psi_{1}+\left[\frac{\delta^{\Delta}}{\delta}+\frac{2 \delta^{\sigma} a_{1} \overline{C_{1}}}{\delta}\right] w_{1}-\frac{\overline{C_{1}} \delta^{\sigma}}{r \delta^{2}} w_{1}^{2}, \quad t \in\left[s_{1}, t_{1}\right] .
\end{aligned}
$$

Then, we have

$$
\psi_{1} \leqslant w_{1}^{\Delta}+\left[\frac{\delta^{\Delta}}{\delta}+\frac{2 \delta^{\sigma} a_{1} \overline{C_{1}}}{\delta}\right] w_{1}-\frac{\overline{C_{1}} \delta^{\sigma}}{r \delta^{2}} w_{1}^{2} .
$$


Multiplying (2.26) by $\left(u^{\sigma}(t)\right)^{2}$ and integrating from $s_{1}$ to $t_{1}$, we have

$$
\begin{aligned}
\int_{s_{1}}^{t_{1}} \psi_{1}(t)\left(u^{\sigma}(t)\right)^{2} \Delta t \leqslant & \int_{s_{1}}^{t_{1}}\left(u^{\sigma}\right)^{2} w_{1}^{\Delta} \Delta t \\
& +\int_{s_{1}}^{t_{1}}\left(u^{\sigma}\right)^{2}\left[\frac{\delta^{\Delta}}{\delta}+\frac{2 \delta^{\sigma} a_{1} \overline{C_{1}}}{\delta}\right] w_{1}(t) \Delta t-\int_{s_{1}}^{t_{1}}\left(u^{\sigma}\right)^{2} \frac{\delta^{\sigma} \overline{C_{1}}}{r \delta^{2}} w_{1}^{2} \Delta t .
\end{aligned}
$$

Using integration by parts, we get

$$
\begin{aligned}
\int_{s_{1}}^{t_{1}} \psi_{1}\left(u^{\sigma}\right)^{2} \Delta t \leqslant & \left.u^{2}(t) w_{1}(t)\right|_{s_{1}} ^{t_{1}}-\int_{s_{1}}^{t_{1}}\left[u+u^{\sigma}\right] u^{\Delta} w_{1}(t) \Delta t \\
& +\int_{s_{1}}^{t_{1}}\left(u^{\sigma}\right)^{2}\left[\frac{\delta^{\Delta}}{\delta}+\frac{2 \delta^{\sigma} a_{1} \overline{C_{1}}}{\delta}\right] w_{1}(t) \Delta t-\int_{s_{1}}^{t_{1}}\left(u^{\sigma}\right)^{2} \frac{\delta^{\sigma} \overline{C_{1}}}{r \delta^{2}} w_{1}^{2} \Delta t .
\end{aligned}
$$

Rearranging and using $u\left(s_{1}\right)=0=u\left(t_{1}\right)$, we get

$$
\begin{aligned}
\int_{s_{1}}^{t_{1}} \psi_{1}\left(u^{\sigma}\right)^{2} \Delta t \leqslant & \int_{s_{1}}^{t_{1}}\left(\left(u^{\sigma}\right)^{2}\left[\frac{\delta^{\Delta}}{\delta}+\frac{2 \delta^{\sigma} a_{1} \overline{C_{1}}}{\delta}\right]-\left[u+u^{\sigma}\right] u^{\Delta}\right) w_{1}(t) \Delta t \\
& -\int_{s_{1}}^{t_{1}}\left(u^{\sigma}\right)^{2} \frac{\delta^{\sigma} \overline{C_{1}}}{r \delta^{2}} w_{1}^{2} \Delta t \\
\leqslant & \int_{s_{1}}^{t_{1}} \frac{r \delta^{2}\left(u^{\sigma}\right)^{2}}{4 \delta_{1}^{\sigma} \overline{C_{1}}}\left(\left[\frac{\delta^{\Delta}}{\delta}+\frac{2 \delta^{\sigma} a_{1} \overline{C_{1}}}{\delta}\right]-\frac{\left[u+u^{\sigma}\right]}{\left(u^{\sigma}\right)^{2}} u^{\Delta}\right)^{2} \Delta t .
\end{aligned}
$$

This implies that

$$
\int_{s_{1}}^{t_{1}}\left[\psi_{1}\left(u^{\sigma}\right)^{2}-\frac{r \delta^{2}\left(u^{\sigma}\right)^{2}}{4 \delta^{\sigma} \overline{C_{1}}}\left(\left[\frac{\delta^{\Delta}}{\delta}+\frac{2 \delta^{\sigma} a_{1} \overline{C_{1}}}{\delta}\right]-\frac{\left[u+u^{\sigma}\right] u^{\Delta}}{\left(u^{\sigma}\right)^{2}}\right)^{2}\right] \Delta t \leqslant 0
$$

which is a contradiction with (2.18). The case $x(t)<0$ on $\left[t_{0}, \infty\right)_{\mathbb{T}}$ is similar, where we use $q(t)>0$ and $g(t) \geqslant 0$ on $\left[s_{2}, t_{2}\right]_{\mathbb{T}}$ and define $w_{2}(t):=\delta(t)\left[\frac{x^{[1]}(t)}{x(t)}+r(t) a_{2}(t)\right]$ for $t \in\left[s_{2}, t_{2}\right]$ to prove that $A_{2}(u) \leqslant 0 ;$ see (2.18). The proof is completed.

From Theorem 2.4, we can establish different sufficient conditions for the oscillation of (1.1) by using different choices of $\delta(t)$ and $a_{i}(t)$ for $i=1,2$. If in Theorem 2.4 we choose $a_{i}(t)$ and $\delta(t)$ such that

$$
a_{i}(t)=-\frac{\delta^{\Delta}(t)}{2 \overline{C_{i}}\left(t, s_{i}\right) \delta^{\sigma}(t)},
$$

we have the following oscillation result for (1.1).

Corollary 2.5. Assume that $(2.3)$ and $\left(\mathrm{H}_{1}\right)$ hold and $\tau(t)>t$. Further assume that there exists an $r d$-continuous function $u \in \mathbb{D}$ such that for $i=1,2$ we have

$$
\int_{s_{i}}^{t_{i}}\left(u^{\sigma}\right)^{2}\left[\varphi_{i}-\frac{r \delta^{2}}{4 \delta^{\sigma} \overline{C_{i}}\left(t, s_{i}\right)}\left(\frac{\left[u+u^{\sigma}\right] u^{\Delta}}{\left(u^{\sigma}\right)^{2}}\right)^{2}\right] \Delta t>0
$$

where $\left.\varphi_{i}:=\delta^{\sigma}\left[Q_{i}-(a r)^{\Delta}+C_{i}\left(t, s_{i}\right) r a^{2}\right)\right]$. Then (1.1) is oscillatory.

Remark 2.6. $\quad$ One can use different values of the functions $a(t)$ and $\delta(t)$ to find new oscillation criteria. The details are left to the interested reader. If $\delta=1$ and $a_{i}=0$, Theorem 2.4 reduces to Corollary 3.4 in [3].

Example 2.7. $\quad$ Let $\mathbb{T}=\mathbb{R}$ and consider the following forced advance differential equation

$$
x^{\prime \prime}(t)+\frac{m^{3} \cos ^{3} t}{\sin ^{4} t\left(1+\cos ^{2} t\right)} x^{3}\left(t+\frac{\pi}{4}\right)=\sin ^{3} t,
$$


where $m$ is a positive parameter. Equation (2.29) is of the form (1.1), where

$$
q(t)=\frac{m^{3} \cos ^{3} t}{\sin ^{4} t\left(1+\cos ^{2} t\right)}, \quad \gamma=3, \quad r(t)=1, \quad g(t)=\sin ^{3} t
$$

We apply Theorem 2.1 to this example. We let

$$
s_{1}=2 n \pi+\frac{3 \pi}{2}, \quad t_{1}=2(n+1) \pi, \quad s_{2}=2 n \pi, \quad t_{2}=2 n \pi+\frac{\pi}{2} .
$$

Note that

$$
q(t)=\frac{m^{3} \cos ^{3} t}{\sin ^{4} t\left(1+\cos ^{2} t\right)} \geqslant 0, \quad \text { and } \quad R(t)=t>0,
$$

on

$$
\left[s_{1}, t_{1}\right) \cup\left[s_{2}, t_{2}\right)=\left[2 n \pi+\frac{3 \pi}{2}, 2(n+1) \pi\right) \cup\left[2 n \pi, 2 n \pi+\frac{\pi}{2}\right),
$$

and

$$
g(t)=\sin ^{3} t\left\{\begin{array}{l}
\leqslant 0: t \in\left[s_{1}, t_{1}\right)=\left[2 n \pi+\frac{3 \pi}{2}, 2(n+1) \pi\right), \\
\geqslant 0: t \in\left[s_{2}, t_{2}\right)=\left[2 n \pi, 2 n \pi+\frac{\pi}{2}\right) .
\end{array}\right.
$$

Here

$$
\begin{aligned}
Q_{1}(t) & :=\gamma(\gamma-1)^{\frac{1}{\gamma}-1}(q(t))^{1 / \gamma} C_{1}\left(t, s_{1}\right)|g(t)|^{1-\frac{1}{\gamma}} \\
& =\frac{3}{2} \sqrt[3]{2} m \delta_{i}(t) \frac{\cos t}{\sin ^{2} t} \sin ^{2} t=\frac{3}{2} \sqrt[3]{2} C_{1}\left(t, s_{1}\right) m \cos t,
\end{aligned}
$$

where

$$
C_{1}\left(t, s_{1}\right)=\frac{R\left(s_{1}\right)-R(\tau(t))}{R\left(s_{1}\right)-R(\sigma(t))}=\frac{2 n \pi+\frac{3 \pi}{2}-\left(t+\frac{\pi}{4}\right)}{2 n \pi+\frac{3 \pi}{2}-t}=\frac{5 \pi-4 t+8 \pi n}{6 \pi-4 t+8 \pi n} .
$$

If we take $u(t)=\sin (4 t)$, then $u\left(s_{i}\right)=u\left(t_{i}\right)=0, u(t) \not \equiv 0$ on $\left[s_{i}, t_{i}\right], i=1,2$, and

$$
\begin{aligned}
A_{1}(u) & :=\int_{2 n \pi+\frac{3 \pi}{2}}^{2(n+1) \pi}\left[r(t)\left(u^{\Delta}(t)\right)^{2}-Q_{i}(t)\left(u^{\sigma}(t)\right)^{2}\right] \Delta t \\
& =\int_{2 n \pi+\frac{3 \pi}{2}}^{2(n+1) \pi}\left[r(t)\left(u^{\Delta}(t)\right)^{2}-Q_{i}(t)\left(u^{\sigma}(t)\right)^{2}\right] d t \\
& =2 \pi-\frac{3}{2} \sqrt[3]{2} m \int_{2 n \pi+\frac{3 \pi}{2}}^{2(n+1) \pi} \cos t \sin ^{2}(4 t)\left(\frac{5 \pi-4 t+8 \pi n}{6 \pi-4 t+8 \pi n}\right) d t .
\end{aligned}
$$

Note that if $m>1$, then for $m$ sufficiently large $A_{1}(u) \leqslant 0$. Similarly for $m$ sufficiently large $A_{2}(u) \leqslant 0$. Hence by Theorem 2.1 we get that if $m$ is sufficiently large, then (2.29) is oscillatory.

\subsection{The case when $\tau(t) \leqslant t$}

In this subsection, we establish some sufficient conditions for oscillation of $(1.1)$ when $\tau(t) \leqslant t$. We introduce the auxiliary function for $i=1,2$,

$$
P_{i}(t):=\gamma(\gamma-1)^{\frac{1}{\gamma}-1}(q(t))^{1 / \gamma} \delta_{i}(t)|g(t)|^{1-\frac{1}{\gamma}},
$$

where

$$
\delta_{i}(t):=\left\{\begin{array}{l}
\frac{r(t) R\left(\tau(t), \tau\left(s_{i}\right)\right)}{r(t) R\left(t, \tau\left(s_{i}\right)\right)+\mu(t)}: \tau(t)<\sigma(t), \\
1: \tau(t)=\sigma(t)
\end{array}\right.
$$

and

$$
R(u, v):=\int_{v}^{u} \frac{1}{r(s)} \Delta s, \quad \text { with } \quad R(u, v)>0 .
$$


We assume that the following condition holds:

$\left(\mathrm{H}_{2}\right)$ Given any $T \in\left[t_{0}, \infty\right)_{\mathbb{T}}$ there exist points $T<s_{i}<t_{i}$ in $\mathbb{T}$ for $i=1,2$ such that $q(t) \geqslant 0$ for $t \in\left[\tau\left(s_{1}\right), t_{1}\right)_{\mathbb{T}} \cup\left[\tau\left(s_{2}\right), t_{2}\right)_{\mathbb{T}}$ and

$$
g(t)\left\{\begin{array}{l}
\leqslant 0: t \in\left[\tau\left(s_{1}\right), t_{1}\right)_{\mathbb{T}}, \\
\geqslant 0: t \in\left[\tau\left(s_{2}\right), t_{2}\right)_{\mathbb{T}} .
\end{array}\right.
$$

Theorem 2.8. Assume that $\left(\mathrm{H}_{2}\right)$ holds and $\tau(t) \leqslant t$. Furthermore, assume that there exists an $r d$-continuous function $u \in \mathbb{D}$ such that for $i=1,2$,

$$
B_{i}(u):=\int_{s_{i}}^{t_{i}}\left[r(t)\left(u^{\Delta}(t)\right)^{2}-P_{i}(t)\left(u^{\sigma}(t)\right)^{2}\right] \Delta t \leqslant 0 .
$$

Then (1.1) is oscillatory.

Proof. Assume that there is a nonoscillatory solution $x$ of (1.1). First, we consider the case $x(t)>0$ and $x(\tau(t))>0$ for $t \geqslant T$. Make the Riccati substitution

$$
w(t):=-\frac{r(t) x^{\Delta}(t)}{x(t)}, \quad t \in[T, \infty)_{\mathbb{T}} .
$$

Then as in the proof of Theorem 2.1, we get

$$
w^{\Delta}=q \frac{x^{\gamma}(\tau)}{x^{\sigma}}-\frac{g}{x^{\sigma}}+\frac{w^{2}}{r-\mu w} .
$$

By assumption, we can choose $s_{1}$ and $t_{1}>T$ such that $q(t) \geqslant 0$ and $g(t) \leqslant 0$ for $t \in\left[s_{1}, t_{1}\right]_{\mathbb{T}}$. From Theorem 2.1, we know that $x^{[1]}(t)$ is strictly decreasing on $\left[s_{1}, t_{1}\right]_{\mathbb{T}}$. Then from (1.1), we have

$$
\left.\left(x^{[1]}\right)^{\Delta}=-q(t) x^{\gamma}(\tau(t))\right)+g(t) \leqslant 0, \quad \text { for } \quad t \in\left[\tau\left(s_{1}\right), t_{1}\right)_{\mathbb{T}} .
$$

Hence $x^{[1]}$ is decreasing for $t \in\left[\tau\left(s_{1}\right), t_{1}\right)_{\mathbb{T}}$. Following the proof of Theorem 2.1, we have for $t \in\left[s_{1}, t_{1}\right)_{\mathbb{T}}$, that

$$
x^{\sigma}(t)-x(\tau(t))=\int_{\tau(t)}^{\sigma(t)} \frac{z(s)}{r(s)} \Delta s \leqslant\left(z(\tau(t)) \int_{\tau(t)}^{\sigma(t)} \frac{1}{r(s)} \Delta s,\right.
$$

and this implies that

$$
\frac{x^{\sigma}(t)}{x(\tau(t))} \leqslant 1+\frac{(z(\tau(t))}{x(\tau(t))} \int_{\tau(t)}^{\sigma(t)} \frac{1}{r(s)} \Delta s .
$$

On the other hand, for $t \in\left[s_{1}, t_{1}\right)_{\mathbb{T}}$, we have that

$$
x(\tau(t))>\int_{\tau\left(s_{1}\right)}^{\tau(t)} \frac{z(s)}{r(s)} \Delta s \geqslant\left(z(\tau(t)) \int_{\tau\left(s_{1}\right)}^{\tau(t)} \frac{1}{r(s)} \Delta s,\right.
$$

which leads to

$$
\frac{z(\tau(t))}{x(\tau(t))}<\left(\int_{\tau\left(s_{1}\right)}^{\tau(t)} \frac{1}{r(s)} \Delta s\right)^{-1}, \quad t \in\left(s_{1}, t_{1}\right)_{\mathbb{T}} .
$$

Using this last inequality and (2.34), we get by [4, Theorems 1.75 and 1.77] that

$$
\begin{aligned}
\frac{x^{\sigma}(t)}{x(\tau(t))} & <1+\frac{\int_{\tau(t)}^{\sigma(t)} \frac{1}{r(s)} \Delta s}{\int_{\tau\left(s_{1}\right)}^{\tau(t)} \frac{1}{r(s)} \Delta s}=\frac{\int_{\tau\left(s_{1}\right)}^{\sigma(t)} \frac{1}{r(s)} \Delta s}{\int_{\tau\left(s_{1}\right)}^{\tau(t)} \frac{1}{r(s)} \Delta s} \\
& =\frac{\int_{\tau\left(s_{1}\right)}^{t} \frac{1}{r(s)} \Delta s+\int_{t}^{\sigma(t)} \frac{1}{r(s)} \Delta s}{\int_{\tau\left(s_{1}\right)}^{\tau(t)} \frac{1}{r(s)} \Delta s} \\
& =\frac{r(t) R\left(t, \tau\left(s_{1}\right)\right)+\mu(t)}{r(t) R\left(\tau(t), \tau\left(s_{1}\right)\right)}=\frac{1}{\delta_{1}(t)}, \quad t \in\left(s_{1}, t_{1}\right)_{\mathbb{T}} .
\end{aligned}
$$


Hence, we get the desired inequality

$$
x(\tau(t)) \geqslant \delta_{1}(t) x^{\sigma}(t), \quad t \in\left(s_{1}, t_{1}\right)_{\mathbb{T}} .
$$

To see that (2.35) holds for $t=s_{1}$ note that if $\tau\left(s_{1}\right)=\sigma\left(s_{1}\right)$, then $\delta_{1}\left(s_{1}\right)=1$ and clearly (2.35) holds. If $\tau\left(s_{1}\right)<\sigma\left(s_{1}\right)$, then $\delta_{1}\left(s_{1}\right)=0$ and so again (2.35) holds for $t=s_{1}$. Substituting (2.35) into (2.33), we get

$$
w^{\Delta} \geqslant q(t)\left(\delta_{1}(t)\right)^{\gamma}\left(x^{\sigma}\right)^{\gamma-1}+\frac{|g(t)|}{x^{\sigma}}+\frac{w^{2}(t)}{r(t)-\mu(t) w(t)} .
$$

Using the fact that the function $G(y):=a y^{\gamma-1}+(b / y)$ satisfies

$$
G(y) \geqslant \gamma(\gamma-1)^{\frac{1}{\gamma}-1} a^{\frac{1}{\gamma}} b^{1-1 / \gamma}, \quad y>0,
$$

we have

$$
\inf _{u>0}\left[q(t)\left(\delta_{1}(t)\right)^{\gamma}\left(x^{\sigma}\right)^{\gamma-1}+\frac{|g|}{x^{\sigma}}\right] \geqslant P_{1}(t) .
$$

Hence $w^{\Delta} \geqslant P_{1}(t)+\left(w^{2} /(r-\mu w)\right)$, for $t \in\left[s_{1}, t_{1}\right]_{\mathbb{T}}$. The reminder of the proof is similar to the proof of Theorem 2.1 and hence is omitted.

From Theorem 2.8, we have the following results immediately.

Corollary 2.9. Assume that $\left(\mathrm{H}_{2}\right)$ holds and $\tau(t) \leqslant t$. Further assume for $\alpha_{i}=\pi^{2} /\left(t_{i}-s_{i}\right)^{2}, i=1,2$ that

$$
F_{i}(u):=\int_{s_{i}}^{t_{i}}\left[r(t) \alpha_{i} \cos ^{2} \sqrt{\alpha_{i}}\left(t-s_{i}\right)-P_{i}(t) \sin ^{2} \sqrt{\alpha_{i}}\left(\sigma(t)-s_{i}\right)\right] \leqslant 0 .
$$

Then (1.1) is oscillatory.

Corollary 2.10. Assume that $\left(\mathrm{H}_{2}\right)$ holds and $\tau(t)<t$. Further assume for $i=1,2$ that

$$
\int_{s_{i}}^{t_{i}}\left[r(t) Z_{i}^{2}-P_{i}(t) H^{2}\left(t_{i}, \sigma(t)\right) H^{2}\left(\sigma(t), s_{i}\right)\right] \Delta t \leqslant 0,
$$

where $Z_{i}=h_{i}\left(t, s_{i}\right) H\left(t_{i}, t\right) \sqrt{H\left(t, s_{i}\right)}+h_{i}\left(t_{1}, t\right) H\left(\sigma(t), s_{i}\right) \sqrt{H\left(t_{i}, t\right)}$. Then (1.1) is oscillatory.

In the following, we employ a generalized Riccati technique to establish new oscillation criteria for (1.1) in the delay case. We define for $i=1,2$,

$$
\eta_{i}(t):=\left\{\begin{array}{l}
\frac{r(t) R\left(t, s_{i}\right)}{r(t) R\left(t, s_{i}\right)+\mu(t)}: \sigma(t)>t \\
1: \sigma(t)=t .
\end{array}\right.
$$

Theorem 2.11. Assume that $\left(\mathrm{H}_{2}\right)$ holds and $\tau(t) \leqslant t$. Further assume that there exist a function $a_{i}(t)$, a positive differentiable function $\delta$, and a $\mathrm{C}_{\mathrm{rd}}^{1}$-function $u$ such that for $i=1,2$, we have

$$
\int_{s_{i}}^{t_{i}}\left(u^{\sigma}\right)^{2}\left[\psi_{i}-\frac{r \delta^{2}}{4 \delta^{\sigma} \eta_{i}}\left(\left[\frac{\delta^{\Delta}+2 \delta^{\sigma} a_{i} \eta_{i}}{\delta}\right]+\frac{\left[u+u^{\sigma}\right] u^{\Delta}}{\left(u^{\sigma}\right)^{2}}\right)^{2}\right] \Delta t \geqslant 0,
$$

where $\psi_{i}(t):=\delta^{\sigma}(t)\left[P_{i}(t)-\left(a_{i}(t) r(t)\right)^{\Delta}+r \eta_{i}(t) a_{i}^{2}(t)\right]$. Then every solution of (1.1) is oscillatory.

Proof. $\quad$ Suppose to the contrary that $x$ is a nonoscillatory solution of (1.1). Then there is a $T \in\left[t_{0}, \infty\right) \mathbb{T}$ such that $x(\tau(t))$ is of one sign on $[T, \infty)_{\mathbb{T}}$. We consider the case where $x(\tau(t))>0$ for all $t \in[T, \infty)_{\mathbb{T}}$. Define the function $w_{1}(t)$ by the generalized Riccati substitution (2.20). Proceeding as in the proof of Theorem 2.1, we get

$$
w_{1}^{\Delta} \leqslant-\delta^{\sigma} P_{1}+\frac{\delta^{\Delta}}{\delta} w_{1}+\delta^{\sigma}\left(r a_{1}\right)^{\Delta}-\delta^{\sigma} r\left(\frac{x^{\Delta}}{x}\right)^{2} \frac{x}{x^{\sigma}} .
$$


From the definition of $w_{1}(t)$, we see that

$$
\left(\frac{x^{\Delta}}{x}\right)^{2}=\left[\frac{w_{1}}{r \delta}-a_{1}\right]^{2}=\left[\frac{w_{1}}{r \delta}\right]^{2}+a_{1}^{2}-2 \frac{w_{1} a_{1}}{r \delta} .
$$

Similar to the proof of (2.35) in Theorem 2.8 by using [4, Theorems 1.75 and 1.77], we get that

$$
x(t) \geqslant \eta_{1}(t) x^{\sigma}(t), \quad \text { for } t \in\left[s_{1}, t_{1}\right)_{\mathbb{T}} .
$$

Substituting from (2.37) and (2.38) into (2.36), we obtain

$$
\begin{aligned}
w_{1}^{\Delta} & \leqslant-\delta^{\sigma} P_{1}+\frac{\delta^{\Delta}}{\delta} w_{1}+\delta^{\sigma}\left(r a_{1}\right)^{\Delta}-\delta^{\sigma} r \eta_{1}\left[\left[\frac{w_{1}}{r \delta}\right]^{2}+a_{1}^{2}-2 \frac{a_{1} w_{1}}{r \delta}\right] \\
& =-\delta^{\sigma} P_{1}+\frac{\delta^{\Delta}}{\delta} w_{1}+\delta^{\sigma}\left(r a_{1}\right)^{\Delta}-\frac{\eta_{1} \delta^{\sigma} w_{1}^{2}}{r \delta^{2}}-\delta^{\sigma} r \eta_{1} a_{1}^{2}+2 \frac{\delta^{\sigma} \eta_{1} a_{1}}{\delta} w_{1} \\
& =-\psi_{1}+\left[\frac{\delta^{\Delta}}{\delta}+\frac{2 \delta^{\sigma} a_{1} \eta_{1}}{\delta}\right] w_{1}-\frac{\eta_{1} \delta^{\sigma}}{r \delta^{2}} w_{1}^{2} .
\end{aligned}
$$

Then, we have

$$
\psi_{1} \leqslant-w_{1}^{\Delta}+\left[\frac{\delta^{\Delta}}{\delta}+\frac{2 \delta^{\sigma} a_{1} \eta_{1}}{\delta}\right] w_{1}-\frac{\eta_{1} \delta^{\sigma}}{r \delta^{2}} w_{1}^{2} .
$$

The reminder of the proof is similar to the proof of Theorem 2.5 and hence is omitted.

From Theorem 2.11, we can establish different sufficient conditions for the oscillation of (1.1) by using different choices of $\delta(t)$ and $a_{i}(t)$ for $i=1,2$. If in Theorem 2.11, we choose $a_{i}(t)$ and $\delta(t)$ such that

$$
a_{i}(t)=-\frac{\delta^{\Delta}(t)}{2 \eta_{i}(t) \delta^{\sigma}(t)},
$$

we have the following oscillation result for (1.1).

Corollary 2.12. Assume that $\left(\mathrm{H}_{2}\right)$ holds and $\tau(t) \leqslant t$. Further assume that there exists an $r d$ continuous function $u \in \mathbb{D}$ such that for $i=1,2$ we have

$$
\int_{s_{i}}^{t_{i}}\left(u^{\sigma}\right)^{2}\left[\psi_{i}-\frac{r \delta^{2}}{4 \delta^{\sigma} \eta_{i}}\left(\frac{\left[u+u^{\sigma}\right] u^{\Delta}}{\left(u^{\sigma}\right)^{2}}\right)^{2}\right] \Delta t \geqslant 0,
$$

where $\left.\psi_{i}:=\delta^{\sigma}\left[P_{i}-\left(a_{i} r\right)^{\Delta}+\eta_{i} r a_{i}^{2}\right)\right]$. Then (1.1) is oscillatory.

Remark 2.13. One can use different values of the functions $a(t)$ and $\delta(t)$ to find new oscillation criteria. The details are left to the interested reader. If $r(t)=1, \delta=1$ and $a_{i}=0$, Theorem 2.11 reduces to Theorem 1 in [30].

Example 2.14. Let $\mathbb{T}=\mathbb{R}$ and consider the following linear forced delay differential equation

$$
x^{\prime \prime}(t)+\frac{m^{3} \cos ^{3} t}{\sin ^{4} t\left(1+\cos ^{2} t\right)} x^{3}\left(t-\frac{\pi}{4}\right)=\sin ^{3} t,
$$

where $m$ is a positive parameter. Equation (2.42) is of the form (1.1) where

$$
q(t)=\frac{m^{3} \cos ^{3} t}{\sin ^{4} t\left(1+\cos ^{2} t\right)}, \quad \gamma=3, \quad r(t)=1, \quad g(t)=\sin ^{3} t .
$$

We apply Theorem 2.8 in this example. We let

$$
s_{1}=2 n \pi+\frac{7 \pi}{4}, \quad t_{1}=2(n+1) \pi, \quad s_{2}=2 n \pi+\frac{\pi}{4}, \quad t_{2}=2 n \pi+\frac{\pi}{2} .
$$

Note that

$$
q(t)=\frac{m^{3} \cos ^{3} t}{\sin ^{4} t\left(1+\cos ^{2} t\right)} \geqslant 0
$$


on

$$
\left[\tau\left(s_{1}\right), t_{1}\right) \cup\left[\tau\left(s_{2}\right), t_{2}\right)=\left[2 n \pi+\frac{3 \pi}{2}, 2(n+1) \pi\right) \cup\left[2 n \pi, 2 n \pi+\frac{\pi}{2}\right),
$$

and

$$
g(t)=\sin t\left\{\begin{array}{l}
\leqslant 0: t \in\left[\tau\left(s_{1}\right), t_{1}\right)=\left[2 n \pi+\frac{3 \pi}{2}, 2(n+1) \pi\right) \\
\geqslant 0: t \in\left[\tau\left(s_{2}\right), t_{2}\right)=\left[2 n \pi, 2 n \pi+\frac{\pi}{2}\right) .
\end{array}\right.
$$

Here

$$
\begin{aligned}
P_{i}(t) & :=\gamma(\gamma-1)^{\frac{1}{\gamma}-1}(q(t))^{1 / \gamma} \delta_{i}(t)|g(t)|^{1-\frac{1}{\gamma}} \\
& =\frac{3}{2} \sqrt[3]{2} m \delta_{i}(t) \frac{\cos t}{\sin ^{2} t} \sin ^{2} t=\frac{3}{2} \sqrt[3]{2} m \delta_{i}(t) \cos t,
\end{aligned}
$$

and

$$
\delta_{1}(t)=\frac{\tau(t)-\tau\left(s_{1}\right)}{t-\tau\left(s_{1}\right)}=\frac{t-2 n \pi-\frac{7 \pi}{4}}{t-2 n \pi-\frac{3 \pi}{2}} .
$$

If we take $u(t)=\sin (4 t)$, then $u\left(s_{i}\right)=u\left(t_{i}\right)=0, u(t) \not \equiv 0$ on $\left[s_{i}, t_{i}\right], i=1,2$, and

$$
\begin{aligned}
B_{1}(u) & :=\int_{2 n \pi+\frac{7 \pi}{4}}^{2(n+1) \pi}\left[r(t)\left(u^{\Delta}(t)\right)^{2}-P_{i}(t)\left(u^{\sigma}(t)\right)^{2}\right] \Delta t \\
& =\int_{2 n \pi+\frac{7 \pi}{4}}^{2(n+1) \pi}\left[r(t)\left(u^{\Delta}(t)\right)^{2}-P_{i}(t)\left(u^{\sigma}(t)\right)^{2}\right] d t \\
& =2 \pi-\frac{3}{2} \sqrt[3]{2} m \int_{2 n \pi+\frac{7 \pi}{4}}^{2(n+1) \pi} \cos t \sin ^{2}(4 t) \frac{t-2 n \pi-\frac{7 \pi}{4}}{t-2 n \pi-\frac{3 \pi}{2}} d t .
\end{aligned}
$$

Note that if $m>1$, then for $m$ sufficiently large $B_{1}(u) \leqslant 0$. Similarly, for $m$ sufficiently large $B_{2}(u) \leqslant 0$. Hence by Theorem 2.8, we get that if $m$ is sufficiently large, then (2.42) is oscillatory.

\section{References}

1 Agarwal R P, Bohner M, Saker S H. Oscillation of second order delay dynamic equation. Can Appl Math Quart, 2005, 13: $1-17$

2 Anderson D R. Oscillation of second-order forced functional dynamic equations with oscillatory potentials. J Differ Equ Appl, 2007, 13: 407-421

3 Anderson D R. Interval criteria for oscillation of nonlinear second-order dynamic equations on time scales. Nonlinear Anal Theor Meth Appl, 2008, 69: 4614-4623

4 Bohner M, Peterson A. Dynamic Equations on Time Scales: An Introduction with Applications. Boston: Birkhäuser, 2001

5 Bohner M, Peterson A. Advances in Dynamic Equations on Time Scales. Boston: Birkhäuser, 2003

6 Bohner M, Saker S H. Oscillation of second order nonlinear dynamic equations on time scales. Rocky Mountain J Math, 2004, 34: 1239-1254

7 Bohner M, Tisdell C. Oscillation and nonoscillation of forced second order dynamic equations. Pacific J Math, 2007, 230: $59-71$

8 Erbe L, Hassan T S, Peterson A, et al. Oscillation criteria for half-linear delay dynamic equations on time scales. Nonlinear Dyn Syst Theor, 2009, 9: 51-68

9 Erbe L, Hassan T S, Peterson A, et al. Oscillation criteria for sublinear half-linear delay dynamic equations on time scales. Int J Differ Equ, 2008, 3: 227-245

10 Erbe L, Peterson A, Saker S H. Oscillation criteria for second-order nonlinear dynamic equations on time scales. J London Math Soc, 2003, 76: 701-714

11 Erbe L, Peterson A, Saker S H. Kamenev-type oscillation criteria for second-order linear delay dynamic equations. Dyn Syst Appl, 2006, 15: 65-78

12 Erbe L, Peterson A, Saker S H. Oscillation criteria for a forced second-order nonlinear dynamic equation. Dyn Equ J Differ Equ Appl, 2008, 14: 997-1009 
13 Erbe L, Hassan T S, Peterson A, et al. Interval oscillation criteria for forced second-order nonlinear delay dynamic equations with oscillatory potential. Dyn Cont Discret Impuls Syst Ser A, 2010, 13: 533-542

14 Güvenilir A F, Zafer A. Second-order oscillation of forced functional differential equations with oscillatory potentials. Comput Math Appl, 2006, 51: 1395-1404

15 Hilger S. Analysis on measure chains - a unified approach to continuous and discrete calculus. Results Math, 1990, 18: $18-56$

16 Huang M, Feng W. Oscillation for forced second-order nonlinear dynamic equations on time scales. Electron J Differ Equ, 2006, 2006: 1-8

17 Huang M, Feng W. Forced Oscillation of second-order nonlinear dynamic equations on time scales. Electron J Qual Theor Differ Equ, 2008, 36: 1-13

18 Kac V, Chueng P. Quantum Calculus. New York: Springer, 2002

19 Nasr A H. Necessary and sufficent conditions for the oscillation of forced nonlinear second order differential equations with delayed argument. J Math Anal Appl, 1997, 212: 51-59

20 Saker S H. Oscillation of second-order nonlinear neutral delay dynamic equations on time scales. J Comp Appl Math, 2005, 177: 375-387

21 Saker S H. Oscillation of second-order forced nonlinear dynamic equations on time scales. Electron J Qual Theor Differ Equ, 2005, 23: 1-17

22 Saker S H. New oscillation criteria for second-order nonlinear dynamic equations on time scales. Nonlinear Funct Anal Appl, 2006, 11: 170-351

23 Saker S H. Oscillation of nonlinear dynamic equations on time scales. Appl Math Comp, 2004, 148: 81-91

24 Saker S H. Oscillation criteria of second-order half-linear dynamic equations on time scales. J Comp Appl Math, 2005, 177: $375-387$

25 Saker S H. Oscillation Theorey of Dynamic Equations on Time Scales, Second and Third Orders. Berlin: Lambert Academic Publishing, 2010

26 Saker S H. Boundedness of solutions of second-order forced nonlinear dynamic equations. Rocky Mount J Math, 2006, 36: 2027-2039

27 Saker S H. Oscillation of second-order forced nonlinear dynamic equations on time scales. Electron J Qual Theor Differ Equ, 2005, 23: 1-17

28 Saker S H. Kamenev-type oscillation criteria for forced Emden-Fowler superlinear difference equations. Electron J Differ Equ, 2002, 2002: 1-9

29 Spedding V. Taming nature's numbers. New Scientist, 2003, 179: 28-31

30 Sun Y G. A note on Nasr's and Wong's papers. J Math Anal Appl, 2003, 286: 363-367

31 Wang Q R. Interval criteria for oscillation of second-order nonlinear differential equations. J Comp Appl Math, 2007, 205: 231-238

32 Wong J S W. Oscillation criteria for a forced second-order linear differential equation. J Math Anal Appl, 1999, 231: 233-240 